\title{
Fingerprint Image Denoising Via the Improved Total Variation (TV)
}

\author{
Algorithm \\ ZHU Rong, WANG Yong, LIU Jingxing \\ School of Information Science and Engineering, Qufu Normal University, Rizhao, Shandong \\ 276826, China \\ Experimental Center, Qufu Normal University, Rizhao, Shandong, 276826, China \\ School of Information Science and Engineering, Qufu Normal University, Rizhao, Shandong \\ 276826, China
}

\begin{abstract}
Keywords: Fingerprint image denoising, total variation, split Bregman iteration, relaxation factors.

Abstract. This paper proposes several improvements for a fingerprint image denoising method that is based on nonlocal total variation (TV) models using split Bregman iteration. The main improvement involves the addition of relaxation factors to the two-step iterative process of split Bregman iterative algorithms to obtain a double relaxation split Bregman iterative algorithm. The improved method is tested using numerous fingerprint images from FVC2004 databases. The experimental results show that the improved double relaxation split Bregman iterative algorithm achieves significantly better performance in terms of the visual subjective evaluation and the quantitative objective evaluation. The method achieves better noise suppression and effectively retains image edge details.
\end{abstract}

\section{Introduction}

Fingerprinting is a common biometric technology for personal identification. Fingerprints are employed as variables of security during voting, examinations, and banking operations and yield the highest individualization rate. Therefore, fingerprint systems have received significant attention from researchers.

Traditional image denoising is implemented in spatial domains and primarily includes mean filtering [1], median filtering [2] and Wiener filtering [3]. A defect of spatial domain methods is that they usually smooth or blur edges in the image during denoising. Conversely, the frequency domain methods that use wavelets frequently obtain more satisfying results. Because wavelets exhibit superior time-frequency characteristics and multi-resolution characteristics, it is extensively applied in the field of image denoising [4, 5]. The frequency-based denoising methods have achieved a better denoising effect. However, defects of suppresses on both noise and other high-frequency features of the image produce an overly smoothed and denoised image.

The total variation (TV) model was proposed by Rudin et al. in 1992[6]. In recent decades, concern with the variational-based denoising model has increased. One of the most classic models is the Rudin-Osher-Fatemi (ROF) model [6] due to its ability to smooth noise without a loss of image edges; it has been successfully applied in many image restoration fields. The model can retain the image edge while removing noises. However, some disadvantages of the model include, such as false edges due to misjudgment of image noise and the regional present ladder effect. 
Due to existing problems of the TV model, some researchers have proposed improved algorithms. The split Bregman method for L1-regularized problems was proposed in [7], and nonlocal total variation models for multiplicative noise removal using the split Bregman iteration were introduced in [8].

This paper proposes several improvements for a fingerprint denoising method that is based on nonlocal total variation models using split Bregman iteration. The main improvement involves the addition of relaxation factors to the two-step iterative process of split Bregman iterative algorithms to obtain a double relaxation split Bregman iterative algorithm.

\section{Methods}

Traditional denoising methods are not sufficient for image edge preserving;denoising causes edges to generate a fuzzy definite effect. In 1992, Rudin, Osher and Fatemi proposed the ROF model [6]. In 2009, the split Bregman method, which was proposed by Osher and Goldstein [7], overcame the shortcomings of the ROF model; it is considered to be more efficient in processing denoising problems.

Considering the anisotropy of the total variation model,

$$
\min _{u}\left\{\left|\nabla_{x} u\right|+\left|\nabla_{y} u\right|+\frac{\mu}{2}\|u-f\|_{2}^{2}\right\}
$$

First, using $d_{x}, d_{y}$ instead of $\nabla_{x} u, \nabla_{y} u$, we obtain the following constraint problem:

$$
\min _{u}\left\{\left|d_{x}\right|+\left|d_{y}\right|+\frac{\mu}{2}\|u-f\|_{2}^{2}\right\}, \text { st. } \quad d_{x}=\nabla_{x} u, d_{y}=\nabla_{y} u
$$

After weakening the constraints and increasing the fidelity term, we obtain

$$
\min _{d_{x}, d_{y}, u}\left\{\left|d_{x}\right|+\left|d_{y}\right|+\frac{\mu}{2}\|u-f\|_{2}^{2}+\frac{\lambda}{2}\left\|d_{x}-\nabla_{x} u\right\|_{2}^{2}+\frac{\lambda}{2}\left\|d_{y}-\nabla_{y} u\right\|_{2}^{2}\right\}
$$

Using the Bregman distance in this formula, to obtain the following format:

$$
\min _{d_{x}, d_{y}, u}\left\{\left|d_{x}\right|+\left|d_{y}\right|+\frac{\mu}{2}\|u-f\|_{2}^{2}+\frac{\lambda}{2}\left\|d_{x}-\nabla_{x} u-b_{x}\right\|_{2}^{2}+\frac{\lambda}{2}\left\|d_{y}-\nabla_{y} u-b_{y}\right\|_{2}^{2}\right\}
$$

Consider sub-problems about $u$ :

$$
u^{k+1}=\min _{u}\left\{\frac{\mu}{2}\|u-f\|_{2}^{2}+\frac{\lambda}{2}\left\|d_{x}^{k}-\nabla_{x} u-b_{x}^{u}\right\|_{2}^{2}+\frac{\lambda}{2}\left\|d_{y}^{k}-\nabla_{y} u-b_{y}^{k}\right\|_{2}^{2}\right\}
$$

The first-order optimality conditions are as follows:

$$
(\mu /-\lambda \Delta) u^{k+1}=\mu f+\lambda \nabla_{x}^{T}\left(d_{x}^{k}-b_{x}^{k}\right)+\lambda \nabla_{y}^{T}\left(d_{y}^{k}-b_{y}^{k}\right)
$$

To achieve optimal efficiency, select the Gauss-Sediel method to obtain an approximate solution for $u$.

$$
u_{i, j}^{k+1}=G_{, j}^{k}
$$




$$
\begin{aligned}
& G_{, j}^{k}=\frac{\lambda}{\mu+4 \lambda}\left(u_{i+1, j}^{k}+u_{i-1, j}^{k}+u_{i, j+1}^{k}+u_{i, j-1}^{k}+d_{x, i-1, j}^{k}-d_{x, i, j}^{k}\right. \\
& \left.+d_{y, i, j-1}^{k}-d_{y, i, j}^{k}-b_{x, i-1, j}^{k}+b_{x, i, j}^{k}-b_{y, i, j-1}^{k}+b_{y, i, j}^{k}\right)+\frac{\mu}{\mu+4 \lambda} f_{i, j}
\end{aligned}
$$

Then, apply the double relaxation split Bregman iterative algorithm to obtain the following double relaxation anisotropy split Bregman total variation denoising algorithms.

\section{Experiment results}

Experiments were performed on various standard gray-scale images from the FVC2004 databases [9]. All experiments were conducted using MATLAB 2014a on a personal computer with an Intel Xeon Core i7-3770 central processing unit and 4 GB of random access memory.

In the denoising experiment, the noise of the standard deviation $\sigma=20$ is added to all test images. Contrastive algorithms include median filters, mean filters, wiener filtering, the wavelet transform, the TV model, the ROF model and the improved total variation (TV) algorithm.

Because a large number of experimental data are consistent with the results, only a few experiment diagrams and test results are provided. The experimental results indicate that only two images were randomly selected from the FVC2004 fingerprint database, i.e., the image names are 101_2.tif in DB1, 101_1.tif in DB2 respectively. Different algorithms for the denoising results of the two images are shown in Figure 1-2.

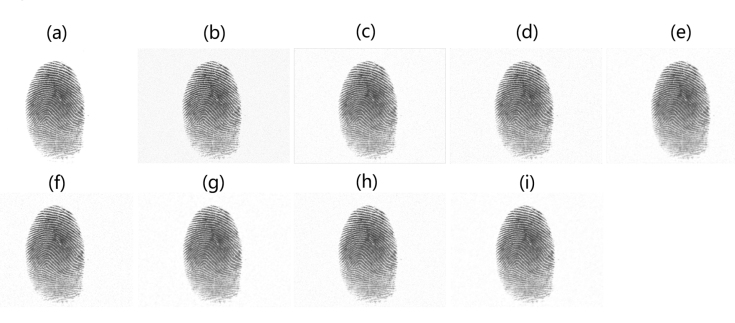

Fig. 1 101_2.tif in DB1. (a) original image; (b) noisy image; (c) result for the median filter; (d) result for the mean filter; (e) result for the W iener filter; (f) result by the wavelet transform; (g) result for the TV model; (h) result for the ROF model; (i) result for the improved TV algorithm.

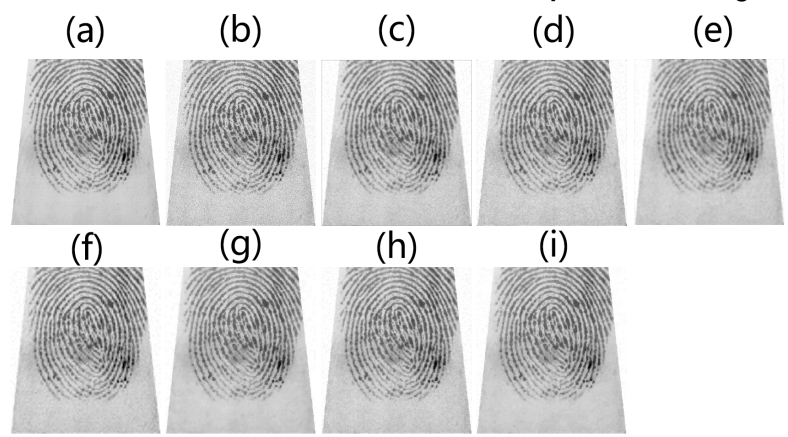

Fig. 2. 101_1.tif in DB2. (a) original image; (b) noisy image; (c) result for the median filter; (d) result for the mean filter; (e) result for the Wiener filter; (f) result by the wavelet transform; $(\mathrm{g})$ result for the TV model; $(\mathrm{h})$ result for the ROF model; (i) result for the improved TV algorithm.

The peak signal-to-noise ratio (PSNR) is the most extensively employed objective measurement and evaluation of image quality. The larger is the PSNR value, the smaller is the image distortion. PSNR [10] is defined as follows:

$$
P S N R=10 \times \log _{10}\left(\frac{\left(2^{n}-1\right)^{2}}{N S E}\right)
$$


where $N S E$ is the mean square error between the original image and the processed image.

Table 1 lists the output PSNR for different algorithms on the test images shown in Figure 1-3. Table 1 summarizes the results of image denoising using the median filters, the mean filters, Wiener filtering, the wavelet transform, TV algorithm, the ROF model and the improved total variation (TV) algorithm. The experiments are realized for six different images in the FVC2004 fingerprint database. The results in Table 1 suggest that the improved total variation (TV) algorithm improves the denoising performance.

Table 1. Output PSNR for different algorithms on the test images shown in Figure 1-Figure 2.

\begin{tabular}{|c|c|c|c|c|c|c|c|c|}
\hline Image & Noisy image & Median & Mean & Wiener & Wavelet & TV & ROF & Improved \\
\hline 101_2.tif in DB1 & 24.1762 & 28.875 & 27.1138 & 28.5366 & 28.925 & 30.1958 & 30.1901 & 30.6262 \\
\hline 101_1.tif in DB2 & 22.3617 & 27.014 & 25.5516 & 26.1667 & 26.5375 & 28.3214 & 28.5194 & 28.6333 \\
\hline
\end{tabular}

\section{Conclusions}

In this paper, several improvements to a fingerprint image denoising method that is based on nonlocal total variation(TV) models using split Bregman iteration have been proposed. The performance of the proposed methods is compared with median filters, mean filters, Wiener filtering, the wavelet transform, the TV model and the ROF model. Using experiments conducted on test images, the proposed method was determined to improve the results of the previously mentioned methods with a slight increase in performance in terms of method noise, visual quality and PSNR. In some cases, the ROF model has demonstrated improved performance when compared to the proposed method.

\section{Acknowledgements}

The study was supported by a Project of the Shandong Province Higher Educational Science and Technology Program (J14LN67), and experimental teaching reform project of Qufu Normal University(sj201419).

This work was supported in part by the NSFC under grant No. 61572284, and Shenzhen Municipal Science and Technology Innovation Council (No.JCYJ20140417172417174).

\section{References}

[1] Coupé P, Yger P, Prima S, et al. An optimized blockwise nonlocal means denoising filter for 3-D magnetic resonance images [J]. Medical Imaging, IEEE Transactions on, 2008, 27(4): 425-441. [2]Chen T, Ma K, Chen L H. Tri-state median filter for image denoising [J]. Image Processing, IEEE Transactions on, 1999, 8(12): 1834-1838.

[3] Ghael S P, Sayeed A M, Baraniuk R G. Improved wavelet denoising via empirical Wiener filtering[C]//Optical Science, Engineering and Instrumentation'97. International Society for Optics and Photonics, 1997: 389-399.

[4] Malfait M, Roose D. Wavelet-based image denoising using a Markov random field a priori model [J]. Image Processing, IEEE Transactions on, 1997, 6(4): 549-565.

[5] Nikpour M, Hassanpour H. Using diffusion equations for improving performance of wavelet-based image denoising techniques [J]. IET image processing, 2010, 4(6): 452-462.

[6] Rudin L I, Osher S, Fatemi E. Nonlinear total variation based noise removal algorithms [J]. Physica D: Nonlinear Phenomena, 1992, 60(1): 259-268.

[7] Goldstein T, Osher S. The split Bregman method for L1-regularized problems [J]. SIAM Journal on Imaging Sciences, 2009, 2(2): 323-343. 
[8] Dong F, Zhang H, Kong D X. Nonlocal total variation models for multiplicative noise removal using split Bregman iteration[J]. Mathematical and Computer Modelling, 2012, 55(3): 939-954.

[9] Mallat S. A wavelet tour of signal processing [M]. Academic press, 1999.

[10] Huynh-Thu Q, Ghanbari M. Scope of validity of PSNR in image/video quality assessment [J]. Electronics letters, 2008, 44(13): 800-801. 